\title{
Brugada syndrome: clinical and genetic findings
}

\author{
Georgia Sarquella-Brugada, MD', Oscar Campuzano, BSc, $\mathrm{PhD}^{2,3}$, \\ Elena Arbelo MD, $\mathrm{PhD}^{4}$, Josep Brugada, $\mathrm{MD}, \mathrm{PhD}^{1,4}$ and Ramon Brugada, MD, PhD ${ }^{2,3,5}$
}

Brugada syndrome is a rare, inherited cardiac disease leading to ventricular fibrillation and sudden cardiac death in structurally normal hearts. Clinical diagnosis requires a Brugada type I electrocardiographic pattern in combination with other clinical features. The most effective approach to unmasking this diagnostic pattern is the use of ajmaline and flecainide tests, and the most effective intervention to reducing the risk of death is the implantation of a cardioverter defibrillator. To date, 18 genes have been associated with the disease, with the voltage-gated sodium channel a type $\mathrm{V}$ gene (SCN5A) being the most common one to date. However, only 30-35\% of diagnosed cases are attributable to pathogenic variants in known genes, emphasizing the need for further genetic studies. Despite recent advances in clinical diagnoses and genetic testing, risk stratification and clinical management of patients with Brugada syndrome remain challenging. Genet Med advance online publication 23 April 2015

Key Words: arrhythmias; Brugada syndrome; genetics; sudden death; syncope
More than 20 years ago, eight individuals were resuscitated from sudden cardiac death (SCD) caused by documented ventricular fibrillation (VF); all showed a characteristic ST segment elevation in the right precordial leads and a structurally normal heart. ${ }^{1}$ In 1996, the term "Brugada syndrome" (BrS) was used to describe what was previously known as "right bundle branch block, persistent ST segment elevation, and sudden death syndrome."2 A year later, BrS was reported to be the same disease as sudden unexplained nocturnal death syndrome (SUNDS). In other countries, BrS has different names: Lai Tai (Thailand), Pokkuri (Japan), and Bangungut (Philippines). Currently, the prevalence of $\mathrm{BrS}$ is estimated at $\sim 3-5$ in 10,000 people, and it is higher in young men of Southeast Asian origin. It is the main cause of death among young healthy men in Southeast Asia. ${ }^{3}$ Recent reports suggest that $\mathrm{BrS}$ could be responsible for $4 \%$ of all SCD and up to $12 \%$ of sudden death in patients with structurally normal hearts. The clinical phenotype manifests in adulthood, at a mean age of 41 years, and it is 8- to 10-times more frequent in males. Frequently, sudden death can be the first manifestation of the disease. ${ }^{4}$ In 1998, the genetic basis of $\mathrm{BrS}$ was established when the sodium channel protein type $\mathrm{V}$ subunit $\alpha$ gene $(S C N 5 A)$, which encodes the $\alpha$-subunit of the voltage-gated $\mathrm{Na}_{\mathrm{v}} 1.5$ cardiac sodium channel responsible for regulating rapid sodium current $\left(\mathrm{I}_{\mathrm{Na}}\right)$, was associated with the disease. $^{5}$

Currently, BrS is recognized as a rare, inherited cardiac channelopathy caused by an alteration of ionic currents that leads to ventricular arrhythmias and SCD. It is clinically characterized by ST segment elevation in leads V1-V3 of the electrocardiogram, but incomplete penetrance and variable expressivity confound the diagnosis. ${ }^{6}$ Despite the identification of 18 associated genes, $65-70 \%$ of clinically diagnosed cases remain without an identifiable genetic cause. ${ }^{4}$

\section{Diagnostic criteria}

\section{CLINICAL DIAGNOSIS}

The clinical diagnosis of BrS requires the identification of the ST segment elevation in the right precordial leads at baseline or after the use of sodium blockers. Three different electrocardiographic (ECG) patterns can be often observed in families with BrS: type I, which consists of a coved-type ST segment elevation greater than $2 \mathrm{~mm}$ followed by a descending negative $\mathrm{T}$ wave in at least two right precordial leads (V1 to V3); type II ST elevation, saddleback-shaped patterns with a high initial increase followed by an ST elevation greater than $2 \mathrm{~mm}$; and saddleback-shaped patterns with a high initial increase followed by an ST elevation less than $2 \mathrm{~mm}$ (Figure 1). The second Brugada Consensus Report proposed that only type I is diagnostic for $\mathrm{BrS}^{7}$ and, in 2013, it was proposed that a definitive diagnosis of BrS considers both spontaneous type I pattern and a provoked type I pattern (with a baseline type II or III pattern) in at least one right precordial lead (V1 or V2). ${ }^{8}$ The diagnosis of BrS is currently accepted in those patients with a type I ECG pattern and any of the following clinical features: documented VF; polymorphic ventricular tachycardia (VT); inducibility of VT with programmed electrical stimulation; family history of SCD at younger than age 45 years; coved-type ECGs in family members; unexplained syncope; or nocturnal agonal respiration

The first two authors contributed equally to this work.

${ }^{1}$ Pediatric Arrhythmia Unit, Hospital Sant Joan de Déu, University of Barcelona, Barcelona, Spain; ${ }^{2}$ Cardiovascular Genetics Centre, University of Girona-IDIBGI, Girona, Spain;

${ }^{3}$ Department of Medical Sciences, School of Medicine, University of Girona, Spain; ${ }^{4}$ Arrhythmia Unit, Hospital Clinic de Barcelona, University of Barcelona, Barcelona, Spain;

${ }^{5}$ Cardiology Service, Hospital Josep Trueta, Girona, Spain. Correspondence: Ramon Brugada (ramon@brugada.org)

Submitted 12 January 2015; accepted 10 February 2015; advance online publication 23 April 2015. doi:10.1038/gim.2015.35 


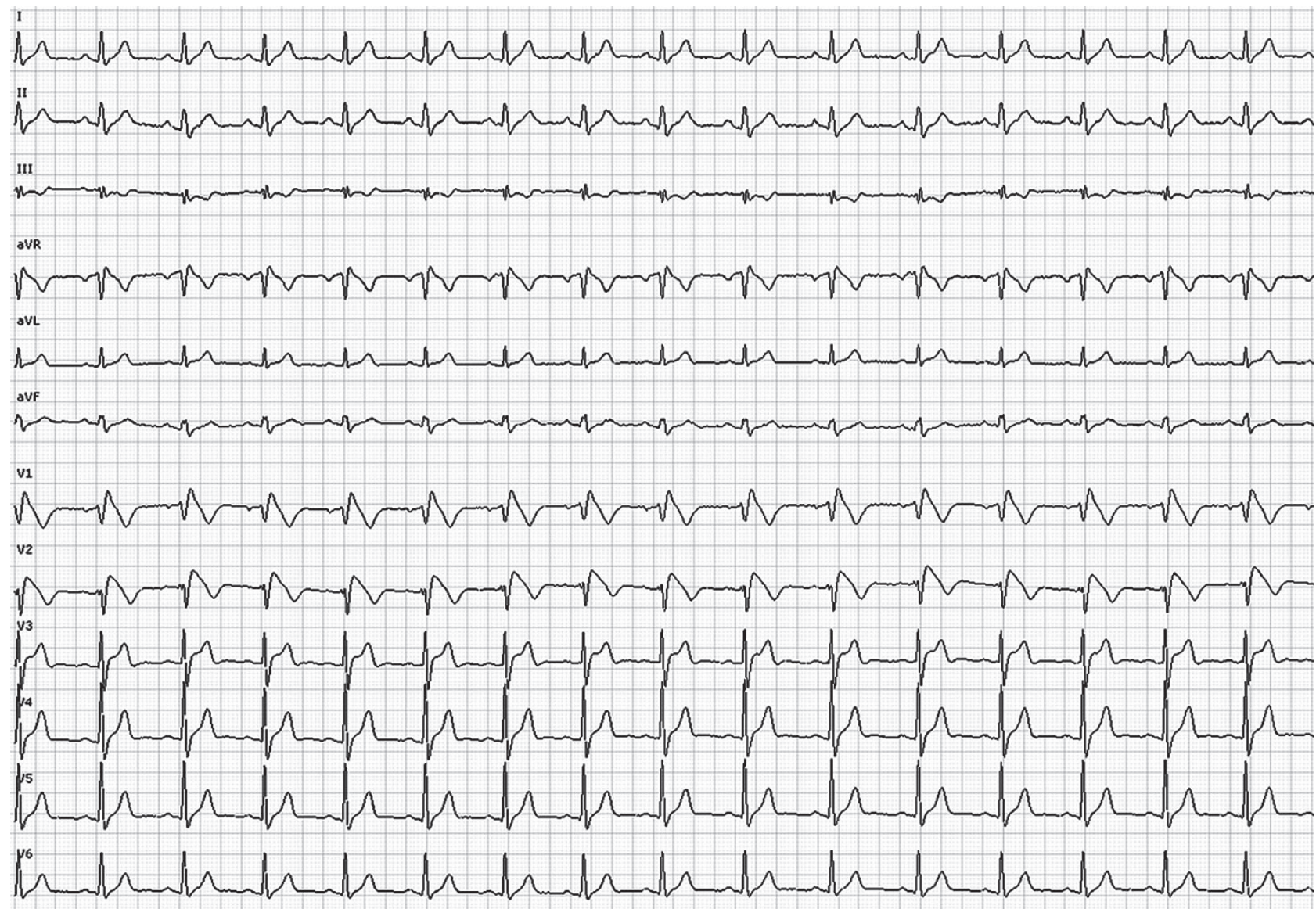

Figure 1 ECG showing a Brugada syndrome (BrS) type I pattern.

(Figure 1). ${ }^{8}$ Both type II and type III patterns are suggestive of BrS, but not diagnostic. A type I ECG pattern is observed in only $25 \%$ of tracings, and most ECG will normalize at followup. Despite that it is suggested that the normalization of the ECG conveys a lower risk, these individuals should continue to receive close follow-up, despite being asymptomatic and with a normal ECG pattern, because they remain at risk for SCD. A type I Brugada ECG pattern is found rarely in the European population, but it appears more commonly in Southeast Asia, with an estimated prevalence of $0.12 \%$ in Japan. ${ }^{9,10}$

No apparent structural heart disease can be found in BrS, at least macroscopically. Some microscopic myocardial alterations have been reported, suggesting that the channelopathy may induce cardiomyopathic changes in some patients. ${ }^{11,12}$ Thus, the presence of important structural alterations should suggest an alternative diagnosis.

\section{Diagnostic tools}

The concealed forms of BrS may be unmasked by drug tests. Indication for these provocation tests are family history of BrS, family history of unexplained sudden death with abnormal ECG or syncope, or symptoms with suspicious ECG abnormalities. Intravenous administration of class IC antiarrhythmic agents, including ajmaline, flecainide, or procainamide, which act as sodium channel blockers, are able to elicit the coved-type ECG diagnostic pattern of $\mathrm{BrS} .{ }^{13}$ An unequal response to intravenous administration of flecainide and ajmaline has been reported in BrS patients, ${ }^{14}$ suggesting that ajmaline is better at unmasking the ECG pattern. This was also observed in families with BrS-associated pathogenic variations in SCN5A, with an ability to detect $80 \%$ of genetic carriers, with the most powerful drug, ajmaline. Because nearly $25 \%$ of drug-induced tests may result in a false-negative result, ${ }^{15}$ a repeat test should be considered. ${ }^{16}$ In a recent report, Conte et al. ${ }^{17}$ repeated ajmaline challenge in a cohort after puberty (older than age 16 years) who previously showed negative drug test (during childhood). They were able to unmask a BrS type I ECG in 23\% of cases, suggesting a potential role for hormonal, autonomic, or epigenetic factors in the ECG response to ajmaline during childhood. This is the first report of changes in the response to ajmaline over time. In addition, it is important to note that time-dependent variability of ECG in BrS could influence interpretation of provocative testing during and after flecainide infusion. Hence, it has been recently reported that those longer periods of ECG recording increase the probability of recognition of the type I ECG. ${ }^{18}$

An alternative tool has been proposed for diagnosing BrS: the full stomach test. ${ }^{19}$ In this test, ECGs are performed before and after a large meal. In BrS patients, this test reveals ST 


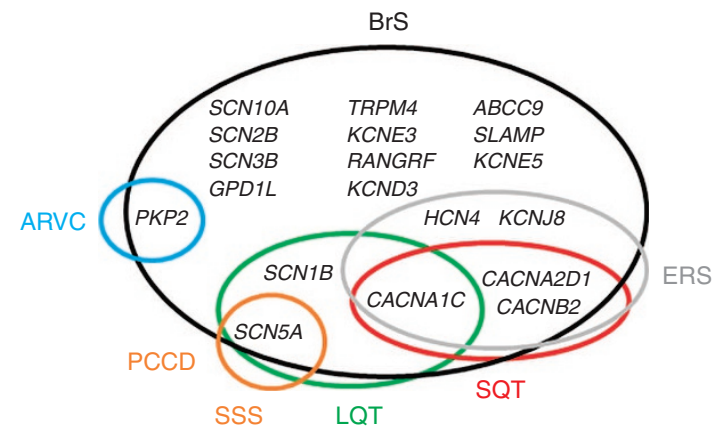

Figure 2 Diagram showing genes associated with Brugada syndrome (BrS) and other overlapping diseases. AF, atrial fibrillation; ARVC, arrhythmogenic right ventricular cardiomyopathy; ERS, early repolarization syndrome; LQT, long QT syndrome; PCCD, progressive cardiac conduction disease; SQT, short QT syndrome; SSS, sick sinus syndrome.

segment changes that appear to be provoked by an enhanced vagal tone. It is well known that further adrenergic stimulation decreases the elevation of the ST segment in BrS patients, whereas vagal stimulation increases it. It is not uncommon to observe ST elevation just after an exercise test in $\mathrm{BrS}$ patients during the vagal phase of the exercise.

\section{Risk stratification}

Accurate identification and treatment of individuals at high risk for SCD is one of the main challenges in the clinical management of BrS patients. The clinical presentation has a wide range. An important percentage of patients may be identified after experiencing syncope or SCD due to malignant ventricular arrhythmias. However, detection of the ECG pattern in asymptomatic individuals is not rare. ${ }^{16}$ Presence of the spontaneous coved-type (type I) ECG pattern is a crucial indicator of potential arrhythmic events in asymptomatic patients. ${ }^{20}$

There are some controversies in the treatment of BrS, especially when dealing with asymptomatic patients. There is an agreement that the presence of symptoms (syncope or aborted sudden death) before diagnosis in combination with a spontaneous type I ECG at baseline constitutes the strongest predictor of risk. ${ }^{21}$ Similarly, it is accepted that male are more associated with cardiac events in follow-up examinations than females, although females are not spared from arrhythmias and sudden death. ${ }^{22}$ Family history of SCD brings a controversy between what data show and the clinician's decision. Despite that data from the FINGER (France, Italy, Netherlands, and Germany) registry suggest that a family history of SCD is not a strong predictor of a patient's risk, ${ }^{23}$ it is not uncommon for the clinician to opt for implanting an implantable cardioverter defibrillator (ICD) in family members with a type I ECG. Noninvasive markers of arrhythmic risk have been assessed in risk stratification, focused on the presence of late potentials on a signal-averaged ECG, which increases the risk of arrhythmic episodes. ${ }^{24}$ In 2012, Priori et al. ${ }^{25}$ published the PRELUDE (programmed electrical stimulation predictive value) registry, a prospective registry designed to assess the predictive accuracy of sustained VT or VF inducibility and to identify additional predictors of arrhythmic events in BrS patients without history of VT/VF. The authors concluded that VT/VF inducibility is not sufficient to identify high-risk patients, whereas the presence of a spontaneous type I ECG, history of syncope, ventricular effective refractory period less than $200 \mathrm{~ms}$, and QRS fragmentation are useful for identification of candidates for a prophylactic ICD. Recently, Okamura et al. ${ }^{26}$ published a retrospective study regarding BrS patients. The authors concluded that syncope, spontaneous type I Brugada ECG, and positive programmed electrical stimulation are important risk factors for SCD and VF in patients with BrS but without previous cardiac arrest. The utility of electrophysiological (EP) testing for risk stratification is controversial, although the most recent consensus statement on risk stratification recommended the use of EP testing in some case scenarios.?

Finally, data outlining the use of genetics in risk stratification are scarce. The aforementioned PRELUDE and FINGER studies in combination with previous published results ${ }^{27,28}$ concluded that BrS patients who carry a pathogenic variant in SCN5A have more pronounced cardiac conduction defects than $\mathrm{BrS}$ patients who are non-carriers of any pathogenic variant in the SCN5A gene. In addition, patients carrying a truncated protein showed more severe conduction disorders but no increased risk of SCD. ${ }^{29}$ In contrast, a meta-analysis of the main BrS databases available reported no different risk of life-threatening arrhythmias in carriers of a pathogenic variant in the SCN5A gene. Taking all data into account, to date, genetic analysis has not been helpful in determining clinical risk stratification. Genetic data are limited and are not presently used for risk stratification.

In summary, few things are clear from the risk stratification data: symptomatic patients are at higher risk than asymptomatic ones; sudden death survivors are at higher risk than patients with syncope; males are at higher risk than females; patients with type I ECG at baseline have a higher risk than those who require class I antiarrhythmics; and asymptomatic patients may also die suddenly. This latter statement is based on the fact that all symptomatic patients with BrS have remained asymptomatic for decades. Thus, at present, the biggest challenge is the detection of these few asymptomatic individuals who will develop symptoms.

\section{Clinical management}

Currently, the ICD is the only effective therapeutic strategy for the prevention of SCD in BrS patients. ${ }^{20}$ ICD implantation is a class 1 indication in patients with $\mathrm{BrS}$ and a history of either ventricular arrhythmias or aborted SCD. Meanwhile, ICD implantation for an asymptomatic patient with a family history of SCD is class III.

Despite its effectiveness, the ICD has several disadvantages, including high cost, potential procedural complications at implantation, multiple device replacements, and psychological effects due to high rates of complications, mainly inappropriate shocks. Recently, it has been reported that therapy using new subcutaneous ICDs may be an effective option because it avoids 


\begin{tabular}{|c|c|c|c|}
\hline Channel & Inheritance & Locus & Gene \\
\hline \multirow[t]{6}{*}{ Sodium } & \multirow[t]{6}{*}{ Autosomal dominant } & 3p21-p24 & SCN5A \\
\hline & & $3 p 22.3$ & GPD1-L \\
\hline & & $19 q 13.1$ & SCN1B \\
\hline & & $11 q 24.1$ & SCN3B \\
\hline & & $11 q 23.3$ & $S C N 2 B$ \\
\hline & & $3 p 22.2$ & SCN10A \\
\hline \multirow{3}{*}{$\begin{array}{l}\text { Sodium- } \\
\text { associated }\end{array}$} & \multirow[t]{3}{*}{ Autosomal dominant } & $17 p 13.1$ & RANGRF \\
\hline & & $3 p 14.3$ & SLMAP \\
\hline & & $12 \mathrm{p} 11.21$ & $P K P 2$ \\
\hline \multirow[t]{6}{*}{ Potassium } & \multirow[t]{5}{*}{ Autosomal dominant } & $12 \mathrm{p} 12.1$ & $A B C C 9$ \\
\hline & & $11 q 13-q 14$ & KCNE3 \\
\hline & & $12 \mathrm{p} 12.1$ & KCNJ8 \\
\hline & & $15 q 24.1$ & HCN4 \\
\hline & & $1 \mathrm{p} 13.2$ & KCND3 \\
\hline & Chromosome X & Xq22.3 & KCNE5 \\
\hline \multirow[t]{4}{*}{ Calcium } & \multirow[t]{4}{*}{ Autosomal dominant } & $2 \mathrm{p} 13.3$ & CACNA1C \\
\hline & & 10p12.33 & $C A C N B 2 B$ \\
\hline & & $7 q 21-q 22$ & CACNA2D1 \\
\hline & & $19 q 13.33$ & TRPM4 \\
\hline
\end{tabular}

problems associated with the placement of an intravascular lead. ${ }^{30}$ Another therapeutic option is pharmacological treatment to rebalance ionic currents. Several drugs have been tested, but only isoproterenol and quinidine are recommended as pharmacological options in $\mathrm{BrS}^{31}$ Isoproterenol has proven to be useful for treatment of electrical storm in BrS. Quinidine has been shown to prevent VF and suppress spontaneous ventricular arrhythmias. Quinidine is recommended for $\mathrm{BrS}$ patients with ICD and multiple shocks when ICD implantation is contraindicated, and for the treatment of supraventricular arrhythmias in the event that other treatments have been contraindicated. ${ }^{32}$ Unfortunately, quinidine remains unavailable in regions where BrS and SUNDS are endemic, such as Southeast Asia. ${ }^{33}$ Finally, radiofrequency catheter ablation has also been postulated as a therapeutic approach in BrS. It has been reported that electrical disconnection of the right ventricular outflow tract can prevent VF inducibility in high-risk populations affected by $\mathrm{BrS} .^{34}$ Although not free of complications, ${ }^{35}$ epicardial ablation has emerged as a potential treatment for BrS.

Regarding family members, all first-degree relatives of patients clinically diagnosed with $\mathrm{BrS}$ should undergo a further clinical screening, including at least a thorough history and an ECG. The recommendation for those patients with normal ECG having had syncope is to undergo a pharmacological provocative test (flecianide, ajmaline, or procainamide) and, if positive, to consider an EP study to rule out any other possible origin for the syncope. For those patients with abnormal ECG suggesting BrS, a provocative pharmacological test should also be performed.

\section{PHENOTYPE MODULATORS}

Recently, genetic and environmental modulators have been identified that play a major role in the dynamic nature of the
ECG and may also be responsible for ST segment elevation. Genetically predisposed patients may develop worse phenotype when exposed to these factors; therefore, the adoption of preventive measures becomes imperative.

Bradycardia and vagal tone may contribute to ST segment elevation and lethal arrhythmia by decreasing calcium currents. ${ }^{36}$ This explains the greater ST segment elevation recorded in vagal settings and the incidence of ventricular arrhythmias at night.

The most well-known environmental factor affecting the BrS phenotype is temperature. Premature inactivation of the $\mathrm{Na}_{v} 1.5$ sodium channel has been shown to be accentuated at higher temperatures. This suggests that febrile states may increase the risk of ventricular arrhythmias, a particularly important trigger factor among the pediatric population. ${ }^{37}$ Thus, fast temperature control is crucial in BrS patients. Similarly, drug-induced BrS have been reported, ${ }^{38}$ such as cocaine overdose. Cocaine is a potent sodium channel blockade that induces myocardial depression, lethal ventricular arrhythmias, and SCD. ${ }^{39}$

Finally, several medications, antidepressants, antiarrhythmics, and anesthetics have been associated with an increased risk of development of ST elevation and even arrhythmias in genetically predisposed individuals. ${ }^{40}$

It has been pointed out that the genetic defect may be associated with a more severe phenotype, i.e., truncated protein with more severe conduction disorders. ${ }^{29}$ In addition, the incomplete penetrance and variable expressivity of BrS have suggested a role of additional genetic factors in the final phenotype. ${ }^{41}$ Thus, several studies have shown that digenic/compound pathogenic variations may cause more severe $\mathrm{BrS}$ phenotypes. ${ }^{42,43}$

A role of common genetic variants (minor allele frequency (MAF) $>1 \%$ ) as modulators of the phenotype is proposed from recent data. Hence, the single nucleotide polymorphism (SNP) p.H558R in the SCN5A gene has been shown to partially restore the $\mathrm{I}_{\mathrm{Na}}$ impaired by other simultaneous $\mathrm{BrS}$ pathogenic variations in the SCN5A gene. ${ }^{44}$ In addition, SNPs located in the $S C N 5 A$ gene promoter region may play a pathophysiologic role in BrS. Six SNPs in the SCN5A gene promoter have been identified and functionally linked to a reduced expression of the $\mathrm{I}_{\mathrm{Na}}$ in the Japanese population. ${ }^{45}$ Genetic modifiers at the epigenetic ${ }^{46}$ and posttranslationa ${ }^{47}$ levels have been suggested as potential modifiers of ionic current and, therefore, of the phenotype.

Finally, gender-specific differences in hormone levels and potential modifiers of the BrS phenotype have been observed. Regression of the typical ECG features has been reported in castrated men, and levels of testosterone appear to be higher in male patients as compared with controls. ${ }^{48}$ Two hypotheses have been suggested for the gender distinction, the sex-related intrinsic differences in ionic currents, and the hormonal influence. It has also been suggested that the interaction of both hormones and ionic currents affects the final phenotype and BrS features. ${ }^{49}$

\section{GENETIC BASIS}

Since the identification of the first gene associated with $\mathrm{BrS}$ in 1998, reports of other families affected by BrS have confirmed 
that the genetic origin of the disease follows an autosomal dominant pattern of inheritance. More than 250 pathogenic variations associated with BrS have been reported in 18 different genes, which primarily encode for sodium, potassium, and calcium channels, or proteins associated with these channels (Figure 2 and Table 1). ${ }^{4}$ Pathogenic variations in genes encoding desmosomal proteins have also been associated with $\mathrm{BrS}^{50,51}$ Recently, an individual diagnosed with $\mathrm{BrS}$ and concomitant conduction system disease had a large-scale deletion of the SCN5A gene. ${ }^{52}$ This copy number variation is the only rearrangement identified as a cause of the disease to date. Despite these ongoing developments in understanding the genetic causes of $\mathrm{BrS}$, only $30-35 \%$ of clinically diagnosed cases are genetically diagnosed, and most of these (25-30\%) result from pathogenic alterations in SCN5A. ${ }^{53}$ SCN5A is responsible for phase 0 of the cardiac action potential, and pathogenic variations result in the inability of the sodium channel to function properly.

The remaining BrS cases are attributable to alterations in one of the other known BrS-associated genes. Many of the $\mathrm{BrS}$-associated genes have a role in regulating sodium channel function. Several pathogenic variations in three genes (SCN1B, $S C N 2 B, S C N 3 B$ ) encoding $\beta$ subunits of the $\mathrm{Na}_{\mathrm{v}} 1.5$ sodium channel have been discovered to modify the function of the channel (increasing or decreasing $\mathrm{I}_{\mathrm{Na}}$ ). ${ }^{54-56}$ SCN10A, a gene encoding the neuronal sodium channel $\mathrm{Na}_{\mathrm{v}} 1.8$, has been shown to modulate SCN5A expression and the electrical function of the heart. ${ }^{57}$ Pathogenic variations in glycerol-3-phosphate dehydrogenase 1-like (GPD1-L) reduce both the surface membrane expression and the inward $\mathrm{I}_{\mathrm{Na}}$ of $\mathrm{Na}_{\mathrm{v}} 1.5 .{ }^{58,59}$ Another gene, RAN guanine nucleotide release factor $(R A N G R F)$, has been reported to impair the trafficking of $\mathrm{Na}_{\mathrm{v}} 1.5$ to the membrane, leading to $\mathrm{I}_{\mathrm{Na}}$ reduction and clinical manifestation of $\mathrm{BrS} .{ }^{60}$ In 2012, Ishikawa et al. ${ }^{61}$ reported pathogenic variations in the sarcolemmal membrane-associated protein (SLMAP) gene, a gene of unknown function that is found at T-tubules and the sarcoplasmic reticulum. SLMAP causes BrS by modulating the intracellular trafficking of the $\mathrm{Na}_{\mathrm{v}} 1.5$ channel. Recently, pathogenic variations in the plakophilin-2 (PKP2) gene were reported to be associated with $\mathrm{BrS} .{ }^{51} \mathrm{PKP} 2$ is the primary gene responsible for arrhythmogenic right ventricular cardiomyopathy (ARVC), a desmosomal disease characterized by fibro-fatty replacement of myocardium leading to SCD in young men, mainly during exercise. Correlation between the loss of expression of PKP2 and reduced $\mathrm{I}_{\mathrm{Na}}$ has been identified in $\mathrm{BrS}$ patients.

Apart from sodium channels, several potassium channels also have been related to BrS. Published reports have identified gain-of-function pathogenic variants in potassium voltage-gated channel, Isk-related family, member 3 (KCNE3), potassium inwardly rectifying channel, subfamily J, member 8 (KCNJ8), potassium voltage-gated channel, Shal-related subfamily, member 3 (KCND3), and potassium voltage-gated channel, Isk-related family, member 5 (KCNE5) that modify potassium channels. A functional role for the MinK-related peptide 2 (MiRP2) protein, encoded by KCNE3, in the modulation of transient outward current $I_{\text {to }}$ in the human heart suggests that pathogenic variations in the KCNE3 gene can underlie the development of BrS. ${ }^{62}$ Gain-of-function pathogenic variants in the KCND3-encoded $\mathrm{K}_{\mathrm{v}} 4.3$ potassium channel are implicated in the pathogenesis and phenotypic expression of $\mathrm{BrS}$, inducing lethal arrhythmia that has been precipitated by a genetically enhanced $\mathrm{I}_{\text {to }}$ current gradient within the right ventricle. ${ }^{63}$ Interestingly, the KCNE5 gene is located on the X chromosome, adding a new pattern of inheritance for BrS. ${ }^{64}$ Hence, pathogenic variations in KCNE5 cause a gain-of-function effect on $\mathrm{I}_{\text {to }}$ current gradients. Similar is the role of adenosine triphosphate (ATP)-sensitive potassium cardiac channels, which consist of the potassium inward-rectifying channel subunit (Kir6.1), encoded by $K C N J 8,{ }^{65}$ and sulfonylurea receptor subunit 2A (SUR2A), encoded by the ATP-binding cassette, subfamily $\mathrm{C}$ member 9 (ABCC9) gene. ${ }^{66}$ Gain-of-function pathogenic variants in $A B C C 9$ induce changes in ATP-sensitive potassium (K-ATP) channels, and, when coupled with lossof-function pathogenic variants in SCN5A, these pathogenic variants may underlie the severe arrhythmic phenotype of $\mathrm{BrS}$. Finally, BrS has also been associated with hyperpolarizationactivated cyclic nucleotide-gated potassium channel 4 (HCN4). HCN4 is found in the sinus node and cells of the cardiac conduction system, and loss-of-function pathogenic variants in the gene are associated with sinus nodal dysfunction. ${ }^{67}$

Several BrS-associated genes have been shown to regulate calcium channels. Pathogenic variations in calcium channel, voltage-dependent, L-type, $\alpha-1 \mathrm{C}$ subunit (CACNA1C) and calcium channel, voltage-dependent, L-type, $\beta$-2B subunit $(C A C N B 2 B)$ cause a loss of calcium channel function. In both of these cases, the phenotype of $\mathrm{BrS}$ has been reported in combination with a shortening of the QT interval in the ECG. ${ }^{68}$ In 2010, the calcium channel, voltage-dependent, L-type, $\alpha-2 / \delta$ subunit 1 (CACNA2D1) gene was reported to be associated with BrS. ${ }^{69}$ The $\alpha-2 / \delta$ subunit of voltage-dependent calcium channels regulates current density and activation/inactivation kinetics of the calcium channel. Finally, pathogenic variations have also been reported in the transient receptor potential melastatin protein number 4 (TRPM4) gene, a calcium-activated nonselective cation channel that is a member of a large family of transient receptor potential genes. ${ }^{70}$ This gene is involved in conduction blocks, and the consequences of pathogenic variations are diverse. Thus, reduction or increase in TRPM4 channel function may reduce the availability of the sodium channel and lead to BrS.

\section{GENETIC TESTING IN BRUGADA SYNDROME}

Recent advances in identifying the genes involved in $\mathrm{BrS}$ have enabled the use of genetic testing in the clinical diagnostic process. These advances have focused on identifying the genetic cause of the disease, which will help detect asymptomatic genetic carriers at risk for SCD. Despite the identification of 18 genes associated with $\mathrm{BrS}$, current guidelines only recommend genetic testing of the SCN5A gene in clinically diagnosed cases, but not in the absence of a diagnostic BrS type I ECG. ${ }^{8}$ These current guidelines that took into 
consideration technological and economic variables are facing the advent of genetic technologies, like next-generation sequencing (NGS), which give the capacity of analysis of many genes (even exomes or genomes) in a shorter time and at reduced cost. ${ }^{71}$ The benefits of identification of a genetic defect are several: confirmation of the disease; identification of non-genetic carriers; adoption of preventive strategies in genetic carriers; offspring decisions, including preimplantational analyses; and others. All these have to be taken into account when deciding whether to perform limited genetic testing, complex testing, or no genetic testing.

One of the main current questions focuses on genetic testing of less common genes associated with BrS. Current guidelines only recommend genetic testing of the main gene associated with $\mathrm{BrS}(S C N 5 A)$. The main reason not to perform the test is the economic issue (in the public systems). Performing a comprehensive genetic test is expensive (despite use of NGS technology) and the probability of pathogenic variant identification is low. This reason will not be an acceptable explanation to that family, especially if there is a recent sudden death. We believe that all families suffering because of $\mathrm{BrS}$ should be further genetically tested.

Thus, genetic testing should be performed when a positive result is identified in an index case to search for pathogenic variants in family members, whatever their ages, to implement recommendations regarding fever control and avoidance of listed drugs (http://www.brugadadrugs.org). Carriers of a pathogenic variation should be followed closely, and risk stratification should be assessed using clinical parameters. It is crucial to note that the presence of the pathogenic variant in a family member does not confirm the presence of the disease in this individual because of incomplete penetrance reported in $\mathrm{BrS}$ families. In contrast, when the family member is not carrying the pathogenic variant identified in the family, it means that person will not suffer from the disease associated with the pathogenic variant studied but we cannot discard the disease due to other genetic variants.

To date, neither whole exome nor genome analysis has been reported for BrS patients. However, in 2011, exome data of healthy populations from the NHLBI GO Exome Sequencing Project were published (http://snp.gs.washington.edu/ EVS/), identifying nearly $10 \%$ of rare variants previously associated with $\mathrm{BrS}$ as highly pathogenic. These data have helped to clarify the potential pathogenic role of the variants in BrS. ${ }^{72}$ The main problems in using NGS technologies are the large amount of data provided and the insufficient experience to translate this information into clinical practice. One of the crucial elements for the correct interpretation of pathogenicity is the genotype-phenotype correlation in families. This leads to the need for each family to be studied separately, analyzing the variations in each relative, and correlating clinical-genetic information. Final decisions should be made by a group consensus based on the experience of each of the members of the working group in each institution dedicated to this purpose.

\section{BRUGADA SYNDROME AND PREGNANCY}

The gender-related differences in the phenotypic expression of BrS have been widely reported, but the basis for gender distinction is not yet fully understood. ${ }^{73}$ During pregnancy, autonomic and hemodynamic alterations occur, and estrogen and progesterone blood levels are reduced during the peripartum period. The largest study of pregnant women with $\mathrm{BrS}$ has been recently published by Rodríguez-Mañero et al. $^{74}$ This study describes a relatively benign course of pregnancy and peripartum period among women with BrS. In addition, only a few cases exhibiting syncope were found, and the presence of syncope during pregnancy did not seem to be related to a worse outcome of the disease in the postpartum or peripartum periods. Nevertheless, the management of pregnant women affected by BrS should be very strict and multidisciplinary, with cooperation between a cardiologist and an anesthesiologist. ${ }^{75}$ Further clinical assessment and follow-up during the pregnant, postpartum, and peripartum periods should be performed, taking into account the favorable maternal and fetal outcomes of disease.

\section{BRUGADA SYNDROME IN CHILDREN}

In the original description of $\mathrm{BrS}$, three of the eight patients were children. ${ }^{1}$ Since then, several isolated cases have been reported, but the prevalence of $\mathrm{BrS}$ in children is not well-established. So far, the largest study of pediatric BrS patients included 30 cases from 13 European institutions. ${ }^{37}$ Therefore, despite progress in understanding $\mathrm{BrS}$, little is known about this disease in the pediatric population.

Clinical diagnosis of $\mathrm{BrS}$ in children is based on the same ECG pattern as adults, taking into account its transiency. One of the main problems in the ECG registry is the optimal positioning of the right precordial leads in children due to the different shape of the chest in a growing body. Despite a whole spectrum of clinical presentations, an episode of syncope associated with typical ECG pattern should alert the cardiologist. Curiously, no male predominance in symptomatic children has been reported, which may be due to lower levels of testosterone. ${ }^{4}$ As mentioned, fever is a triggering factor for ventricular arrhythmias in BrS patients, including children, in which the increased temperature may unmask the ECG pattern. ${ }^{76}$ Hence, it is recommended that a 12-lead ECG test should be performed during a febrile episode. Moreover, because febrile convulsions are a relatively common occurrence in childhood, ECG should be considered as a part of the diagnostic routine when a febrile seizure occurs.

A few approaches have been studied for treatment of $\mathrm{BrS}$ in children. A sodium channel blocker test (ajmaline $1 \mathrm{mg} / \mathrm{kg}$ or flecainide $2 \mathrm{mg} / \mathrm{kg}$ treatment over $10 \mathrm{~min}$ ) should be restricted to children with a normal baseline ECG and typical symptoms with a positive family history. A spontaneous type I ECG pattern is enough to diagnose $\mathrm{BrS}$ in children. ${ }^{77}$ The existence of an age-dependent response to ajmaline challenge is an intriguing recent finding that might have relevant clinical implications. ${ }^{78}$ However, repeating ajmaline challenge after puberty in patients with an initial negative drug test remains controversial and 
should be further investigated. Controversy also exists regarding whether to perform electrophysiologic study testing in children. When indicated, the protocol remains the same for children as for the adult population. ${ }^{22}$ For a pharmacological approach, hydroquinidine has been shown to be a good alternative to ICD implantation in children who are at risk; further follow-up studies are needed to confirm this finding. ${ }^{79}$ However, children presenting syncope with spontaneous type I ECG are clearly at high risk for SCD and ICD should be considered, irrespective of age.

\section{BRUGADA SYNDROME IN OLDER INDIVIDUALS}

The fourth decade of life is the mean age of clinical manifestations of BrS, mainly in men. So far, only two studies describe clinical features of BrS in aged populations. ${ }^{80}$ Thus, the clinical course and prognosis of BrS in older individuals are unknown. Recently, Conte at al. ${ }^{80}$ published a systematic analysis of $\mathrm{BrS}$ in the aging population, reporting a benign prognosis and lower risk category for BrS patients in comparison to younger patients. Consequently, older patients presented less ventricular arrhythmias and less family history of SCD. However, two main challenges remain controversial: use of drug-induced tests and device-guided management. Thus, despite Conte and colleagues' reporting in the same aforementioned study that "BrS was diagnosed after ajmaline challenge in $86 \%$ of elderly patients," the value of unmasking a type I ECG as well as its safety have not been methodically assessed. Regarding the use of an ICD, a consensus conference reported that older BrS patients with syncope should undergo ICD implantation if life expectancy is at least 6 months. ${ }^{7}$ However, clinical decisions regarding both controversies should be analyzed on a case-by-case basis.

\section{OVERLAPPING SYNDROMES}

The genetic heterogeneity of inherited arrhythmia disorders often show overlapping EP features. ${ }^{81}$ These overlapping syndromes include BrS, early repolarization syndrome (ERS), progressive cardiac conduction disease (PCCD), and other diseases leading to ventricular arrhythmias and syncope/SCD (Figure 2). The array of phenotypes exhibited in these syndromes, even within family members, is mainly due to pathogenic variants in the SCN5A gene encoding for the $\mathrm{Na}_{\mathrm{v}} 1.5$ cardiac sodium channel, which are responsible for the majority of $\mathrm{I}_{\mathrm{Na}}$ in myocytes. ${ }^{82}$

\section{Early repolarization syndrome}

Early repolarization syndrome (ERS) is characterized by J-point elevation, ST segment elevation with upper concavity, and prominent $\mathrm{T}$ waves in at least two contiguous leads. ${ }^{83}$ ERS and BrS share similar ECG tracings, although the degree to which ERS and BrS may overlap remains uncertain. ${ }^{84}$ Several loss-of-function pathogenic variants in the CACNA1C, $C A C N B 2$, and CACNA2D1 genes and gain-of-function pathogenic variants in the KCNJ8 gene have been associated with ERS. Recently, a pathogenic variant in the SCN5A gene has also been identified in patients affected by ERS. ${ }^{35}$ It is unclear whether ER confers a higher risk in patients with inherited arrhythmia syndrome, especially because it is not an uncommon finding in young healthy individuals.

\section{Progressive cardiac conduction disease}

Progressive cardiac conduction disease (PCCD), also called Lev-Lenègre syndrome, is a rare disease characterized by disruption of the cardiac conduction system through degeneration of the His-Purkinje system. This disease is associated with syncope and even SCD. ${ }^{85}$ The presence of PCCD in BrS families is not infrequent, because both diseases result from a reduction in $\mathrm{I}_{\mathrm{Na}}$ and PCCD has been described as a different expression of the $\mathrm{BrS}$ genetic phenotype. Finally, it has been reported that several pathogenic variations in the SCN5A gene are associated with both PCCD and BrS. ${ }^{86}$

\section{Sick sinus syndrome}

Sick sinus syndrome (SSS) is characterized by persistent inappropriate sinus bradycardia, sinus arrest, atrial standstill, and tachycardia-bradycardia syndrome, all of which are associated with dysfunction of the sinoatrial node. ${ }^{87} \mathrm{~A}$ wide range of symptoms has been reported in patients, including syncope and even SCD. The course of SSS can be intermittent and unpredictable, depending on the severity of the underlying heart disease. So far, several pathogenic variations in SCN5A have been associated with SSS. In addition, BrS and SSS were both identified within a single family to be caused by a loss-of-function phenotype in $\mathrm{I}_{\mathrm{Na}} \cdot 8$

\section{Long QT syndrome type III}

Long QT syndrome (LQTS) is an inherited arrhythmogenic disease characterized by prolongation of the QT interval, leading to ventricular tachyarrhythmias, syncope, and even SCD. ${ }^{89}$ Several subtypes of LQTS have been reported, such as type III (LQTS-3) caused by pathogenic variations in the SCN5A gene and responsible for nearly $10 \%$ of all LQTS cases. ${ }^{90}$ Therefore, SCN5A pathogenic variants associated with LQTS induce gain-of-function in contrast to pathogenic variants associated with $\mathrm{BrS}$ (inducing loss-of-function). It remains to be understood how a pathogenic variation in the same gene may induce almost opposite electrical defects. The electrical defect may be determined by the biophysical properties of the altered channel, co-inherited genetic variations, gender, ethnicity, or even other environmental factors.

\section{Atrial fibrillation}

Atrial fibrillation (AF) is the most frequent arrhythmia diagnosed in clinical practice. It is characterized by a chaotic electrical activity of atria leading to irregular ventricular rates. The ECG shows an absence of $\mathrm{P}$ waves and irregularities in the $\mathrm{R}-\mathrm{R}$ intervals. AF requires pharmacological treatment even though the response is highly unpredictable. This fact is due mainly to limited knowledge of the molecular pathophysiology of $\mathrm{AF}^{91,92}$ In the original description of $\mathrm{BrS}$, two of the patients also exhibited AF. ${ }^{1}$ Currently, nearly $30 \%$ of BrS patients carrying a pathogenic variant in the SCN5A gene also show AF with 
evidence of PCCD and prolonged atrioventricular and atrialHis conduction. ${ }^{7.93}$

\section{CONCLUSIONS}

$\mathrm{BrS}$ is an important hereditary cardiac disease leading to ventricular arrhythmias and SCD. Mechanistic processes responsible for variable expressivity and incomplete penetrance remain to be clarified, impeding proper clinical diagnosis, risk stratification, and management. Genetic background is crucial to understanding both the cause of the disease and the variable phenotype. Elucidating the exact mechanisms underlying $\mathrm{BrS}$ and the precise genetic characteristics of each patient are essential to personalizing therapies. So far, the ICD is the most effective therapeutic option, despite several pharmacological approaches that are also currently being used. Combined preclinical, clinical, and comprehensive genetic studies in large cohorts will be indispensable for improving the current guidelines to diagnose $\mathrm{BrS}$, stratify the risk of SCD, and prevent lethal episodes.

\section{DISCLOSURE}

The authors declare no conflict of interest.

\section{REFERENCES}

1. Brugada P, Brugada J. Right bundle branch block, persistent ST segment elevation and sudden cardiac death: a distinct clinical and electrocardiographic syndrome. A multicenter report. J Am Coll Cardio/ 1992;20:1391-1396.

2. Miyazaki T, Mitamura H, Miyoshi S, Soejima K, Aizawa Y, Ogawa S. Autonomic and antiarrhythmic drug modulation of ST segment elevation in patients with Brugada syndrome. J Am Coll Cardiol 1996;27:1061-1070.

3. Nademanee K, Veerakul G, Nimmannit S, et al. Arrhythmogenic marker for the sudden unexplained death syndrome in Thai men. Circulation 1997;96: 2595-2600.

4. Brugada R, Campuzano O, Sarquella-Brugada G, Brugada J, Brugada P. Brugada syndrome. Methodist Debakey Cardiovasc J 2014;10:25-28.

5. Chen Q, Kirsch GE, Zhang D, et al. Genetic basis and molecular mechanism for idiopathic ventricular fibrillation. Nature 1998;392:293-296.

6. Brugada P, Brugada J, Roy D. Brugada syndrome 1992-2012: 20 years of scientific excitement, and more. Eur Heart J 2013;34:3610-3615.

7. Antzelevitch C, Brugada P, Borggrefe M, et al. Brugada syndrome: report of the second consensus conference: endorsed by the Heart Rhythm Society and the European Heart Rhythm Association. Circulation 2005;111:659-670.

8. Priori SG, Wilde AA, Horie M, et al. HRS/EHRA/APHRS expert consensus statement on the diagnosis and management of patients with inherited primary arrhythmia syndromes: document endorsed by HRS, EHRA, and APHRS in May 2013 and by ACCF, AHA, PACES, and AEPC in June 2013. Heart Rhythm 2013;10:1932-1963.

9. Gallagher MM, Forleo GB, Behr ER, et al. Prevalence and significance of Brugada-type ECG in 12,012 apparently healthy European subjects. Int J Cardiol 2008;130:44-48.

10. Miyasaka Y, Tsuji H, Yamada K, et al. Prevalence and mortality of the Brugadatype electrocardiogram in one city in Japan. J Am Coll Cardiol 2001;38: 771-774.

11. Frustaci A, Priori SG, Pieroni M, et al. Cardiac histological substrate in patients with clinical phenotype of Brugada syndrome. Circulation 2005;112: 3680-3687.

12. Frustaci A, Russo MA, Chimenti C. Structural myocardial abnormalities in asymptomatic family members with Brugada syndrome and SCN5A gene mutation. Eur Heart J 2009:30:1763.

13. Morita H, Zipes DP, Wu J. Brugada syndrome: insights of ST elevation, arrhythmogenicity, and risk stratification from experimental observations. Heart Rhythm 2009;6(suppl 11):S34-S43.

14. Wolpert C, Echternach C, Veltmann C, et al. Intravenous drug challenge using flecainide and ajmaline in patients with Brugada syndrome. Heart Rhythm 2005;2:254-260.
15. Hong K, Brugada J, Oliva A, et al. Value of electrocardiographic parameters and ajmaline test in the diagnosis of Brugada syndrome caused by SCN5A mutations. Circulation 2004;110:3023-3027.

16. Berne P, Brugada J. Brugada syndrome 2012. Circ J 2012;76:1563-1571.

17. Conte G, Dewals W, Sieira J, et al. Drug-induced brugada syndrome in children: clinical features, device-based management, and long-term follow-up. J Am Coll Cardiol 2014;63:2272-2279.

18. Calvo D, Rubín JM, Pérez D, et al. Time-dependent responses to provocative testing with flecainide in the diagnosis of Brugada syndrome. Heart Rhythm 2015;12:350-357.

19. Ikeda T, Abe A, Yusu S, et al. The full stomach test as a novel diagnostic technique for identifying patients at risk of Brugada syndrome. J Cardiovasc Electrophysiol 2006;17:602-607.

20. Mashar M, Kwok AJ, Pinder R, Sabir I. The Brugada syndrome revisited. Trends Cardiovasc Med 2014;24:191-196.

21. Priori SG, Napolitano C, Gasparini M, et al. Natural history of Brugada syndrome: insights for risk stratification and management. Circulation 2002;105: 1342-1347.

22. Brugada J, Brugada R, Brugada P. Determinants of sudden cardiac death in individuals with the electrocardiographic pattern of Brugada syndrome and no previous cardiac arrest. Circulation 2003;108:3092-3096.

23. Probst $V$, Veltmann $C$, Eckardt $L$, et al. Long-term prognosis of patients diagnosed with Brugada syndrome: Results from the FINGER Brugada Syndrome Registry. Circulation 2010;121:635-643.

24. Huang Z, Patel C, Li W, et al. Role of signal-averaged electrocardiograms in arrhythmic risk stratification of patients with Brugada syndrome: a prospective study. Heart Rhythm 2009;6:1156-1162.

25. Priori SG, Gasparini M, Napolitano C, et al. Risk stratification in Brugada syndrome: results of the PRELUDE (PRogrammed ELectrical stimUlation preDictive valuE) registry. J Am Coll Cardio/ 2012;59:37-45.

26. Okamura $\mathrm{H}$, Kamakura $\mathrm{T}$, Morita $\mathrm{H}$, et al. Risk stratification in patients with brugada syndrome without previous cardiac arrest. Circ J 2015;79: 310-317.

27. Smits JP, Eckardt L, Probst V, et al. Genotype-phenotype relationship in Brugada syndrome: electrocardiographic features differentiate SCN5A-related patients from non-SCN5A-related patients. J Am Coll Cardio/ 2002;40:350-356.

28. Eckardt L, Probst V, Smits JP, et al. Long-term prognosis of individuals with right precordial ST-segment-elevation Brugada syndrome. Circulation 2005;111: 257-263.

29. Meregalli PG, Tan HL, Probst $\mathrm{V}$, et al. Type of SCN5A mutation determines clinical severity and degree of conduction slowing in loss-of-function sodium channelopathies. Heart Rhythm 2009;6:341-348.

30. Rowley CP, Lobodzinski SS, Gold MR. The subcutaneous defibrillator. Curr Treat Options Cardiovasc Med 2012;14:550-557.

31. Zipes DP, Camm AJ, Borggrefe M, et al.; American College of Cardiology/ American Heart Association Task Force; European Society of Cardiology Committee for Practice Guidelines; European Heart Rhythm Association and the Heart Rhythm Society. ACC/AHA/ESC 2006 guidelines for management of patients with ventricular arrhythmias and the prevention of sudden cardiac death-executive summary: A report of the American College of Cardiology/ American Heart Association Task Force and the European Society of Cardiology Committee for Practice Guidelines (Writing Committee to Develop Guidelines for Management of Patients with Ventricular Arrhythmias and the Prevention of Sudden Cardiac Death) Developed in collaboration with the European Heart Rhythm Association and the Heart Rhythm Society. Eur Heart J 2006;27: 2099-2140.

32. Schweizer PA, Becker R, Katus HA, Thomas D. Successful acute and long-term management of electrical storm in Brugada syndrome using orciprenaline and quinine/quinidine. Clin Res Cardio/ 2010;99:467-470.

33. Viskin S, Wilde AA, Guevara-Valdivia ME, et al. Quinidine, a life-saving medication for Brugada syndrome, is inaccessible in many countries. J Am Coll Cardio/ 2013;61:2383-2387.

34. Nademanee K, Veerakul G, Chandanamattha P, et al. Prevention of ventricular fibrillation episodes in Brugada syndrome by catheter ablation over the anterior right ventricular outflow tract epicardium. Circulation 2011;123:1270-1279.

35. Li A, Behr ER. Brugada syndrome: an update. Future Cardiol 2013;9:253-271.

36. Yan GX, Antzelevitch C. Cellular basis for the Brugada syndrome and other mechanisms of arrhythmogenesis associated with ST-segment elevation. Circulation 1999;100:1660-1666.

37. Probst V, Denjoy I, Meregalli PG, et al. Clinical aspects and prognosis of Brugada syndrome in children. Circulation 2007;115:2042-2048. 
38. Tarradas A, Selga E, Beltran-Alvarez P, et al. A novel missense mutation, 1890T, in the pore region of cardiac sodium channel causes Brugada syndrome. PLoS One 2013;8:e53220.

39. Kolecki PF, Curry SC. Poisoning by sodium channel blocking agents. Crit Care Clin 1997;13:829-848.

40. Vernooy K, Sicouri S, Dumaine R, et al. Genetic and biophysical basis for bupivacaine-induced ST segment elevation and VTNF. Anesthesia unmasked Brugada syndrome. Heart Rhythm 2006;3:1074-1078.

41. Giudicessi JR, Ackerman MJ. Determinants of incomplete penetrance and variable expressivity in heritable cardiac arrhythmia syndromes. Trans/ Res 2013;161:1-14

42. Makita N, Mochizuki N, Tsutsui H. Absence of a trafficking defect in R1232W/ T1620M, a double SCN5A mutant responsible for Brugada syndrome. Circ J 2008;72:1018-1019.

43. Cordeiro JM, Barajas-Martinez H, Hong K, et al. Compound heterozygous mutations P336L and I1660V in the human cardiac sodium channel associated with the Brugada syndrome. Circulation 2006;114:2026-2033.

44. Lizotte $E$, Junttila MJ, Dube MP, et al. Genetic modulation of brugada syndrome by a common polymorphism. J Cardiovasc Electrophysio/ 2009;20:1137-1141.

45. Bezzina CR, Shimizu W, Yang P, et al. Common sodium channel promoter haplotype in asian subjects underlies variability in cardiac conduction. Circulation 2006;113:338-344.

46. Park JK, Martin LJ, Zhang X, Jegga AG, Benson DW. Genetic variants in SCN5A promoter are associated with arrhythmia phenotype severity in patients with heterozygous loss-of-function mutation. Heart Rhythm 2012;9:1090-1096.

47. Beltran-Alvarez P, Pagans S, Brugada R. The cardiac sodium channel is post-translationally modified by arginine methylation. J Proteome Res 2011;10:3712-3719.

48. Matsuo K, Akahoshi M, Seto S, Yano K. Disappearance of the Brugada-type electrocardiogram after surgical castration: a role for testosterone and an explanation for the male preponderance. Pacing Clin Electrophysio/ 2003;26(7 Pt 1):1551-1553.

49. Shimizu W, Matsuo K, Kokubo Y, et al. Sex hormone and gender difference-role of testosterone on male predominance in Brugada syndrome. J Cardiovasc Electrophysio/ 2007;18:415-421.

50. Cerrone M, Delmar M. Desmosomes and the sodium channel complex: implications for arrhythmogenic cardiomyopathy and Brugada syndrome. Trends Cardiovasc Med 2014;24:184-190.

51. Cerrone M, Lin X, Zhang M, et al. Missense mutations in plakophilin-2 cause sodium current deficit and associate with a Brugada syndrome phenotype. Circulation 2014;129:1092-1103.

52. Eastaugh $\amalg$, James PA, Phelan DG, Davis AM. Brugada syndrome caused by a large deletion in SCN5A only detected by multiplex ligation-dependent probe amplification. J Cardiovasc Electrophysiol 2011;22:1073-1076.

53. Kapplinger JD, Tester DJ, Alders M, et al. An international compendium of mutations in the SCN5A-encoded cardiac sodium channel in patients referred for Brugada syndrome genetic testing. Heart Rhythm 2010;7:33-46.

54. Watanabe H, Koopmann TT, Le Scouarnec S, et al. Sodium channel $\beta 1$ subunit mutations associated with Brugada syndrome and cardiac conduction disease in humans. J Clin Invest 2008;118:2260-2268.

55. Riuró H, Beltran-Alvarez $P$, Tarradas $A$, et al. A missense mutation in the sodium channel $\beta 2$ subunit reveals $S C N 2 B$ as a new candidate gene for Brugada syndrome. Hum Mutat 2013:34:961-966.

56. Hu D, Barajas-Martinez $H$, Burashnikov $E$, et al. A mutation in the beta 3 subunit of the cardiac sodium channel associated with Brugada ECG phenotype. Circ Cardiovasc Genet 2009;2:270-278.

57. Hu D, Barajas-Martínez H, Pfeiffer R, et al. Mutations in SCN10A are responsible for a large fraction of cases of Brugada syndrome. J Am Coll Cardiol 2014;64:66-79.

58. Makiyama T, Akao M, Haruna $Y$, et al. Mutation analysis of the glycerol-3 phosphate dehydrogenase-1 like (GPD1L) gene in Japanese patients with Brugada syndrome. Circ J 2008;72:1705-1706.

59. Valdivia CR, Ueda K, Ackerman MJ, Makielski JC. GPD1L links redox state to cardiac excitability by PKC-dependent phosphorylation of the sodium channel SCN5A. Am J Physiol Heart Circ Physiol 2009;297:H1446-H1452.

60. Olesen MS, Holst AG, Svendsen JH, Haunsø S, Tfelt-Hansen J. SCN1Bb R214Q found in 3 patients: 1 with Brugada syndrome and 2 with lone atrial fibrillation. Heart Rhythm 2012;9:770-773.

61. Ishikawa T, Sato A, Marcou CA, et al. A novel disease gene for Brugada syndrome: sarcolemmal membrane-associated protein gene mutations impair intracellular trafficking of hNav1.5. Circ Arrhythm Electrophysiol 2012;5: 1098-1107.
62. Delpón E, Cordeiro JM, Núñez L, et al. Functional effects of KCNE3 mutation and its role in the development of Brugada syndrome. Circ Arrhythm Electrophysio/ 2008;1:209-218.

63. Giudicessi JR, Ye D, Tester DJ, et al. Transient outward current (I(to)) gain-offunction mutations in the KCND3-encoded Kv4.3 potassium channel and Brugada syndrome. Heart Rhythm 2011;8:1024-1032.

64. Ohno S, Zankov DP, Ding WG, et al. KCNE5 (KCNE1L) variants are novel modulators of Brugada syndrome and idiopathic ventricular fibrillation. Circ Arrhythm Electrophysiol 2011;4:352-361.

65. Barajas-Martínez H, Hu D, Ferrer T, et al. Molecular genetic and functional association of Brugada and early repolarization syndromes with $\$ 422 \mathrm{~L}$ missense mutation in KCNJ8. Heart Rhythm 2012:9:548-555.

66. Hu D, Barajas-Martínez H, Terzic A, et al. ABCC9 is a novel Brugada and early repolarization syndrome susceptibility gene. Int J Cardio/ 2014;171:431-442.

67. Ueda K, Hirano Y, Higashiuesato Y, et al. Role of HCN4 channel in preventing ventricular arrhythmia. J Hum Genet 2009;54:115-121.

68. Antzelevitch C, Pollevick GD, Cordeiro JM, et al. Loss-of-function mutations in the cardiac calcium channel underlie a new clinical entity characterized by ST-segment elevation, short QT intervals, and sudden cardiac death. Circulation 2007:115:442-449.

69. Burashnikov E, Pfeiffer R, Barajas-Martinez $\mathrm{H}$, et al. Mutations in the cardiac L-type calcium channel associated with inherited J-wave syndromes and sudden cardiac death. Heart Rhythm 2010;7:1872-1882.

70. Stallmeyer B, Zumhagen S, Denjoy I, et al. Mutational spectrum in the $\mathrm{Ca}(2+)$ activated cation channel gene TRPM4 in patients with cardiac conductance disturbances. Hum Mutat 2012:33:109-117.

71. Buermans HP, den Dunnen JT. Next generation sequencing technology: Advances and applications. Biochim Biophys Acta 2014;1842: 1932-1941.

72. Risgaard $B$, Jabbari $R$, Refsgaard $L$, et al. High prevalence of genetic variants previously associated with Brugada syndrome in new exome data. Clin Genet 2013:84:489-495

73. Tadros R, Ton AT, Fiset C, Nattel S. Sex differences in cardiac electrophysiology and clinical arrhythmias: epidemiology, therapeutics, and mechanisms. Can J Cardiol 2014;30:783-792.

74. Rodríguez-Mañero M, Casado-Arroyo R, Sarkozy A, et al. The clinical significance of pregnancy in Brugada syndrome. Rev Esp Cardiol (Engl Ed) 2014;67:176-180.

75. Giambanco L, Incandela D, Maiorana A, Alio W, Alio L. Brugada syndrome and pregnancy: highlights on antenatal and prenatal management. Case Rep Obstet Gynecol 2014;2014:531648.

76. Dumaine $R$, Towbin JA, Brugada $\mathrm{P}$, et al. Ionic mechanisms responsible for the electrocardiographic phenotype of the Brugada syndrome are temperature dependent. Circ Res 1999:85:803-809.

77. Sorgente A, Sarkozy A, De Asmundis C, et al. Ajmaline challenge in young individuals with suspected Brugada syndrome. Pacing Clin Electrophysiol 2011;34:736-741.

78. Conte G, Brugada P. The challenges of performing ajmaline challenge in children with suspected Brugada syndrome. Open Heart 2014;1:e000031.

79. Probst $V$, Evain $S$, Gournay $V$, et al. Monomorphic ventricular tachycardia due to Brugada syndrome successfully treated by hydroquinidine therapy in a 3year-old child. J Cardiovasc Electrophysio/ 2006;17:97-100.

80. Conte G, DE Asmundis C, Sieira J, et al. Clinical characteristics, management, and prognosis of elderly patients with Brugada syndrome. J Cardiovasc Electrophysio/ 2014;25:514-519.

81. Gray B, Semsarian C, Sy RW. Brugada syndrome: a heterogeneous disease with a common ECG phenotype? J Cardiovasc Electrophysio/ 2014;25:450-456.

82. Kyle JW, Makielski JC. Diseases caused by mutations in Nav1.5 interacting proteins. Card Electrophysiol Clin 2014;6:797-809.

83. Rezus C, Floria M, Moga VD, et al. Early repolarization syndrome: electrocardiographic signs and clinical implications. Ann Noninvasive Electrocardiol 2014;19:15-22

84. Talib AK, Sato N, Kawabata N, et al. Repolarization characteristics in early repolarization and brugada syndromes: insight into an overlapping mechanism of lethal arrhythmias. J Cardiovasc Electrophysio/ 2014;25:1376-1384.

85. Baruteau AE, Probst $V$, Abriel $H$. Inherited progressive cardiac conduction disorders. Curr Opin Cardiol 2015:30:33-39.

86. Probst V, Allouis M, Sacher F, et al. Progressive cardiac conduction defect is the prevailing phenotype in carriers of a Brugada syndrome SCN5A mutation. J Cardiovasc Electrophysiol 2006;17:270-275.

87. Semelka M, Gera J, Usman S. Sick sinus syndrome: a review. Am Fam Physician 2013;87:691-696 


\section{REVIEW ARTICLE}

88. Shimizu N, Iwamoto M, Nakano Y, et al. Long-term electrocardiographic followup from childhood of an adult patient with Brugada syndrome associated with sick sinus syndrome. Circ J 2009;73:575-579.

89. Schwartz PJ, Ackerman MJ, George AL Jr, Wilde AA. Impact of genetics on the clinical management of channelopathies. J Am Coll Cardiol 2013;62:169-180.

90. Nakaya H. SCN5A mutations associated with overlap phenotype of long QT syndrome type 3 and Brugada syndrome. Circ J 2014;78:1061-1062.
91. Tucker NR, Ellinor PT. Emerging directions in the genetics of atrial fibrillation. Circ Res 2014;114:1469-1482.

92. Darbar D, Roden DM. Genetic mechanisms of atrial fibrillation: impact on response to treatment. Nat Rev Cardiol 2013;10:317-329.

93. Morita H, Kusano-Fukushima K, Nagase $\mathrm{S}$, et al. Atrial fibrillation and atrial vulnerability in patients with Brugada syndrome. J Am Coll Cardiol 2002;40:1437-1444. 